\title{
Corrigendum: Evaluation of Glymphatic System Using Diffusion MR Technique in T2DM Cases
}

\author{
Guangwei Yang ${ }^{1}$, Nan Deng ${ }^{2}$, Yi Liu ${ }^{1}$, Yingjiang Gu ${ }^{1}$ and Xiang Yao ${ }^{3 *}$ \\ ${ }^{1}$ Hospital (T.C.M) Affiliated to Southwest Medical University, Luzhou, China, ${ }^{2}$ Luzhou People's Hospital, Luzhou, China, \\ ${ }^{3}$ Department of Radiology, Xiang'an Hospital of Xiamen University, Xiamen, China
}

Keywords: glymphatic system, type 2 diabetes mellitus, MRI, diffusion tensor, perivascular space

\section{A Corrigendum on}

Evaluation of Glymphatic System Using Diffusion MR Technique in T2DM Cases

by Yang, G., Deng, N., Liu, Y., Gu, Y., and Yao, X. (2020). Front. Hum. Neurosci. 14:300. doi: 10.3389/fnhum.2020.00300

\section{OPEN ACCESS}

Approved by:

Frontiers Editorial Office,

Frontiers Media SA, Switzerland

${ }^{*}$ Correspondence:

Xiang Yao

yaoxiang5804@163.com

Specialty section:

This article was submitted to

Brain Imaging and Stimulation,

a section of the journal

Frontiers in Human Neuroscience

Received: 12 October 2020

Accepted: 16 October 2020

Published: 26 January 2021

Citation:

Yang G, Deng N, Liu Y, Gu Y and Yao X (2021) Corrigendum: Evaluation

of Glymphatic System Using Diffusion

MR Technique in T2DM Cases.

Front. Hum. Neurosci. 14:616400.

doi: 10.3389/fnhum.2020.616400
In the original article, there was a mistake in the legend for Figure 1 as published. The figure, which was previously published in the article cited below from the Japanese Journal of Radiology, was reused without appropriate acknowledgment in our article. The correct legend appears below.

Figure 1 The concept of the diffusion tensor image analysis along with the perivascular space (DTI-ALPS) method for perivascular diffusion. (A) The DTI superimposed color display shows the distribution of projection fibers ( $\mathrm{z}$-axis: blue), association fibers ( $\mathrm{y}$-axis: green), and subcortical fibers (x-axis: red). Three regions of interest (ROIs) were placed in the area with projection fibers (projection area), association fibers (association area), and subcortical fibers (subcortical area) to measure the diffusivities in three directions ( $x, y$, and $z$ ). (B) Schematic diagram showing the relationship between the direction of the perivascular space (gray cylinder) and the direction of the fibers. Note that the direction of the perivascular space is perpendicular to the projection and the association fibers. The figure has been reprinted with permission from Taoka et al. (2017).

The authors apologize for this error and state that this does not change the scientific conclusions of the article in any way. The original article has been updated.

\section{REFERENCES}

Taoka, T., Masutani, Y., Kawai, H., Nakane, T., Matsuoka, K., Yasuno, F., et al. (2017). Evaluation of glymphatic system activity with the diffusion MR technique: diffusion tensor image analysis along the perivascular space (DTI-ALPS) in Alzheimer's disease cases. Jpn. J. Radiol. 35, 172-178. doi: 10.1007/s11604-017-0617-z

Copyright (๑ 2021 Yang, Deng, Liu, Gu and Yao. This is an open-access article distributed under the terms of the Creative Commons Attribution License (CC BY). The use, distribution or reproduction in other forums is permitted, provided the original author(s) and the copyright owner(s) are credited and that the original publication in this journal is cited, in accordance with accepted academic practice. No use, distribution or reproduction is permitted which does not comply with these terms. 